\title{
Constipation and Postprandial Pain in a Patient With Shortness of Breath
}

\author{
Sunayana Chopra Pydah, MBA, MHA, MPAM, PA-C; Rohit Gupta; James Evans, PA-C; and \\ SreyRam Kuy, MD, MHS
}

\section{Incidental image findings may be important for resolving seemingly unrelated symptoms at presentation.}

A 62-year-old male veteran with a history of pulmonary embolism (PE) and prostate cancer status after brachytherapy presented to the emergency department with new onset shortness of breath and left-sided chest pain after prolonged car travel. He underwent a chest computed tomography (CT) angiogram that showed no PE recurrence; however, the scan revealed an incidental transverse colon mass that appeared well circumscribed, homogeneous, and radiolucent with no enhancement, septations, or hypervascularity but no evidence of colonic distension or obstruction (Figure 1).

The patient reported having chronic constipation and a dull, left-sided abdominal discomfort for the past year. He noted that his abdominal pain worsened after eating and mildly improved after taking castor oil. He had no surgical history and no family history of cancer. The patient reported no fever, fatigue, weight loss, chills, nausea, vomiting, diarrhea, hematochezia, dysuria, hematuria, or melena. Vital signs, physical examination, and initial routine laboratory work were all within appropriate ranges, and a fecal occult blood test was negative.

What is your diagnosis?

How would you treat this patient?
A colonoscopy was performed, revealing a near-obstructing submucosal mass in the transverse colon near the splenic flexure with a smooth surface and a positive Cushion (Pillow) sign (Figure 2). The patient underwent surgical exploration that resulted in finding a soft, 11-cm lesion arising from the mesenteric side of the transverse colon (Figure 3). Hematoxylin and eosin (H\&E) stains were used on a sample from the mass (Figure 4).

The tumor was enucleated via a colotomy over the mass, and the colotomy repaired primarily. Gross examination revealed homogenous yellow fatty tissue, and the H\&E stains showed mature, well-differentiated adipocytes with uniform nuclei surrounded by a fibrous capsule. Based on this pathologic examination, this patient was diagnosed with a lipoma of the transverse colon. The resected tissue showed negative margins, indicating full removal of the lipoma.

The patient stabilized well after surgery and remained under inpatient care for observation; due to lack of appetite following the surgery, the patient did not start eating solids again until 2 days after the lipoma removal. By postoperative day 4 , the patient had return of bowel function and was tolerating a regular diet with no recurrence of his prandial pain, shortness of breath, or left-sided chest pain. While the precise cause of the patient's initial presentation of shortness of breath and left-sided chest pain was not ascertained, it is likely that the lipoma, near completely obstructed his bowel, caused abdominal contents and distended intestines to push against his diaphragm, leading to pain and dyspnea. This was likely exacerbated by sensitization
Sunayana Chopra Pydah is a Certified Physician Assistant at Santa Clara Valley Medical Center in San Jose, California. Rohit Gupta is a Medical Student at Baylor College of Medicine in Houston, Texas. James Evans is a Physician Assistant at the Michael E. DeBakey Veterans Affairs (VA) Medical Center in Houston, Texas. SreyRam Kuy is an Assistant Professor of Surgery at Baylor College of Medicine and Deputy Chief Medical Officer for the VA Veterans Integrated Services Network 16. Correspondence: Sunayana Chopra Pydah (sunayanapydah @gmail.com)

Fed Pract. 2021;38(7). Published online July 9. doi:10.12788/fp.0154
FIGURE 1 Computed Tomography Showing a Splenic Flexure Colon Mass 
FIGURE 2 Colonoscopy Demonstrating a 11-cm Submucosal Smooth-Textured Transverse Colon Mass

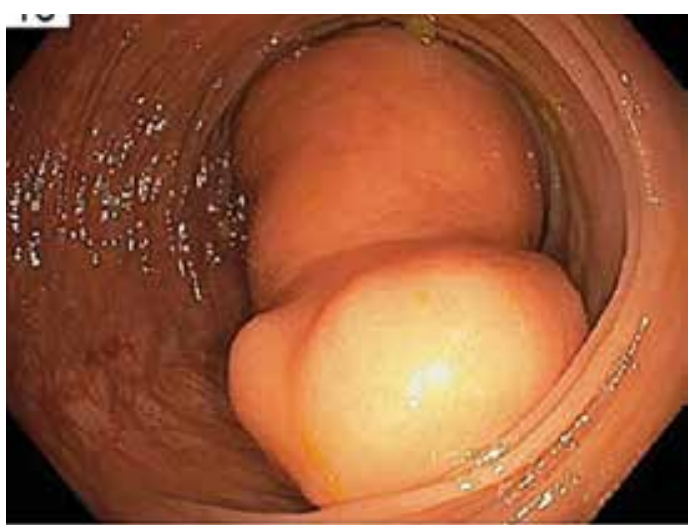

FIGURE 3 Surgical Intraoperative Findings of a Soft Lesion on the Mesenteric Side of the Transverse Colon

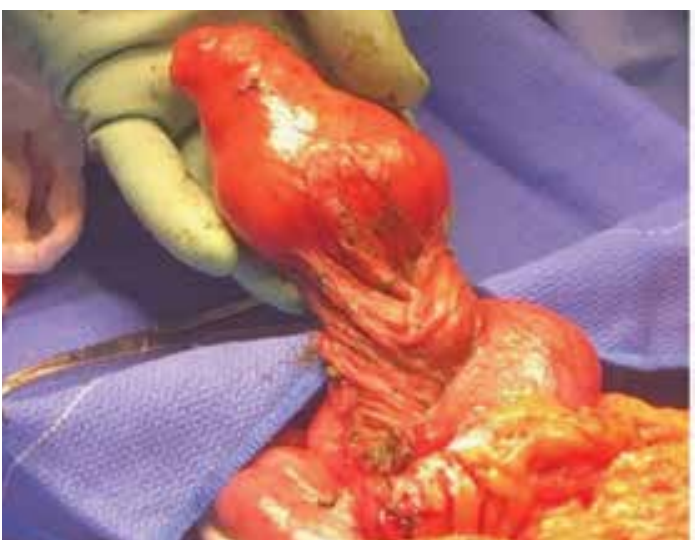

FIGURE 4 Hematoxylin and Eosin Stains, $100 \times$ Magnification

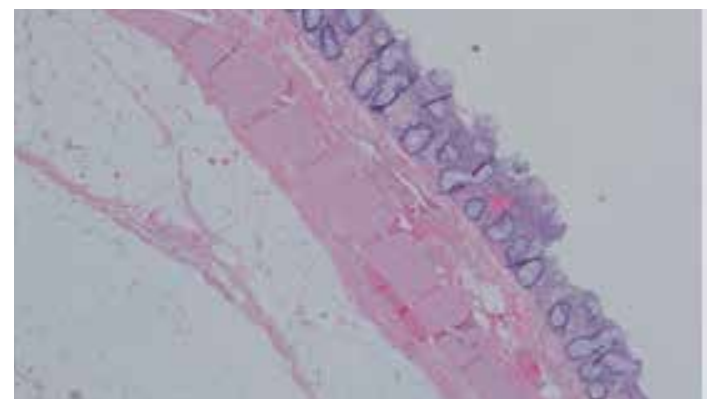

to these symptoms from his prior PE. He was discharged home on postoperative day 4 with outpatient follow-up with general surgery.

\section{DISCUSSION}

Lipomas are common benign tumors arising from aberrantly multiplying adipocytes.
Although lipomas are most commonly found subcutaneously, the lesions can occur anywhere along the gastrointestinal (GI) tract, most often in the colon. ${ }^{1}$ The incidence rate of colon lipomas ranges from 0.2 to $4.4 \%$ among patients in their fifth to sixth decades of life, more commonly found in females. ${ }^{2}$ These lesions are the most common submucosal mesenchymal lesions of the colon, with a predilection for the right ascending colon. ${ }^{1}$ The etiology of colon lipomas is largely unknown; one known cause is trauma, thought to induce cytokine release or HMGA2-LPP fusion gene arrangements leading to adipocyte proliferation. $^{3}$

Most colon lipomas are asymptomatic and discovered incidentally; symptoms typically arise when the lesions are $>2 \mathrm{~cm}$ in diameter and include abdominal pain, changes in bowel habits, rectal bleeding, and in extreme cases, obstruction and perforation. ${ }^{4}$ On CT imaging, colon lipomas will appear radiolucent, homogenous, and well circumscribed. The lesions usually do not warrant intervention unless they are symptomatic. If symptomatic, resection of the lesion is the first-line treatment and usually results in complete resolution of symptoms with no recurrence. ${ }^{2}$

While either a surgical or endoscopic approach may be used for resection, an increased risk of perforation of the colon with larger lipomas has been shown with endoscopic excision. ${ }^{5}$ With surgical resection, an open or minimally invasive approach may be offered, based on surgeon comfort with minimally invasive colon procedures. Minimally invasive colonic surgeries may be associated with a shorter length of stay, decreased postoperative pain, and faster return of bowel function. In this case, the surgeon chose an open approach due to the large size of the mass $(11 \mathrm{~cm})$ as well as location of the mass in the transverse colon, which made it easy to access directly through a small laparotomy incision made in the superior midline over the transverse colon.

When a colonic mesenchymal mass is seen on colonoscopy, it is important to consider other, nonbenign lesions that present this way. The most common malignant mesenchymal tumor of the GI tract is a gastrointestinal stromal tumor 
(GIST), a soft-tissue sarcoma that occurs predominantly in the stomach and small intestine. ${ }^{6}$ These tumors arise from the interstitial cells of Cajal (ICC) and are associated with mutations of KIT and PDGFR- $\alpha$ genes. ${ }^{7}$ The incidence in the United States is approximately 0.70 per 100,000 people per year, predominantly found in adults in their fifth or sixth decade of life. ${ }^{8}$ While this tumor typically occurs in the upper GI tract, very rarely, GISTs can be found in the colon. ${ }^{6}$ Common constitutional symptoms of colon GIST are similar to those of colon lipomas and include abdominal pain, changes in bowel habits, nausea, vomiting, and in some cases, weight loss.

CT imaging is often enough to differentiate a colon lipoma from a colon GIST. On CT, large GIST tumors tend to show irregular, lobulated margins, mucosal ulceration, central necrosis, cavitation, hemorrhage, and hypervascularity-vastly different from the CT findings of colon lipomas. If imaging is equivocal, an ultrasound-guided fine needle aspiration biopsy may be performed, differentiating GIST through the presence of ICC tumor cells as well as KIT and PDGFR- $\alpha$ proteins.

In our patient, colonoscopy showed a positive Cushion sign (tumor indented on depression with biopsy forceps), pathognomonic for a colon lipoma, and CT imaging showed a radiolucent, well-circumscribed lesion. ${ }^{9}$ This was more consistent with a colon lipoma than a GIST. Because the patient was symptomatic with a near obstructing lesion, the appropriate next step was removal of the lesion. Had this instead been a GIST tumor, a more extensive oncologic surgical resection would have been warranted, with adequate mesentery and lymph nodes collected.

This case is notable because colon lipomas exceeding $2 \mathrm{~cm}$ are rare and are usually an incidental finding on CT. However, larger lipomas can lead to symptoms, including obstruction if not removed in a timely manner.

\section{Author disclosures}

The authors report no actual or potential conflicts of interest with regard to this article.

\section{Disclaimer}

The opinions expressed herein are those of the authors and do not necessarily reflect those of Federal Practitioner, Frontline Medical Communications Inc., the US Government, or any of its agencies.

\section{References}

1. Nallamothu G, Adler DG. Large colonic lipomas. Gastroenterol Hepatol (NY). 2011;7(7):490-492.

2. Crocetti $D$, Sapienza $P$, Sterpetti $A V$, et al. Surgery for symptomatic colon lipoma: a systematic review of the literature. Anticancer Res. 2014;34(11):6271-6276.

3. Italiano A, Ebran N, Attias R, et al. NFIB rearrangement in superficial, retroperitoneal, and colonic lipomas with aberrations involving chromosome band 9p22. Genes Chromosomes Cancer. 2008;47(11):971-977. doi:10.1002/gcc.20602

4. Agrawal A, Singh KJ. Symptomatic intestinal lipomas: our experience. Med J Armed Forces India. 2011;67(4):374376. doi:10.1016/S0377-1237(11)60090-7

5. Kim GW, Kwon Cl, Song SH, et al. Endoscopic resection of giant colonic lipoma: case series with partial resection. Clin Endosc. 2013;46(5):586-590. doi:10.5946/ce.2013.46.5.586

6. Reddy RM, Fleshman JW. Colorectal gastrointestinal stromal tumors: a brief review. Clin Colon Rectal Surg. 2006;19(2):69-77. doi:10.1055/s-2006-942347

7. Shinomura Y, Kinoshita K, Tsutsui S, Hirota S. Pathophysiology, diagnosis, and treatment of gastrointestinal stromal tumors. J Gastroenterol. 2005;40(8):775-780. doi:10.1007/s00535-005-1674-0

8. Patel N, Benipal B. Incidence of gastrointestinal stromal tumors in the United States from 2001-2015: a United States cancer statistics analysis of 50 states. $\mathrm{Cu}$ reus. 2019;11(2):e4120. Published 2019 Feb 22. doi:10.7759/cureus.4120

9. Kyawzaw K, Emmanuel O, Sandar L,2 Febin J,Naing LA, Madhavi R. Pillow sign in colonoscopy. MOJ Clin Med Case Rep. 2018;8(2):57-58. doi:10.15406/ mojcr.2018.08.00240 